\title{
Class of PPT bound entangled states associated to almost any set of pure entangled states
}

\author{
Marco Piani ${ }^{1, *}$ and Caterina E. Mora ${ }^{2, \dagger}$ \\ 1 Institute of Theoretical Physics and Astrophysics, University of Gdańsk, 80-952 Gdańsk, Poland \\ ${ }^{2}$ Institut für Quantenoptik und Quanteninformation der Österreichischen Akademie der Wissenschaften, Innsbruck, Austria
}

\begin{abstract}
We analyze a class of entangled states for bipartite $d \otimes d$ systems, with $d$ non-prime. The entanglement of such states is revealed by the construction of canonically associated entanglement witnesses. The structure of the states is very simple and similar to the one of isotropic states: they are a mixture of a separable and a pure entangled state whose supports are orthogonal. Despite such simple structure, in an opportune interval of the mixing parameter their entanglement is not revealed by partial transposition nor by the realignment criterion, i.e. by any permutational criterion in the bipartite setting. In the range in which the states are Positive under Partial Transposition (PPT), they are not distillable; on the other hand, the states in the considered class are provably distillable as soon as they are Nonpositive under Partial Transposition (NPT). The states are associated to any set of more than two pure states. The analysis is extended to the multipartite setting. By an opportune selection of the set of multipartite pure states, it is possible to construct mixed states which are PPT with respect to any choice of bipartite cuts and nevertheless exhibit genuine multipartite entanglement. Finally, we show that every $k$-positive but not completely positive map is associated to a family of nondecomposable maps.
\end{abstract}

PACS numbers:

\section{INTRODUCTION}

Entanglement is a resource required in many task typical of the fields of quantum information and quantum computation [1, 2], like quantum teleportation [3] and superdense coding [4]. While there is a clear definition of what an entangled state is [5], it is in general difficult to determine whether a given state is entangled or not. Correspondingly, the structure of the space of states, as classified with respect to the entanglement property, is still a central issue of investigation. Moreover, in the multipartite case the picture is even more complicated, since it appears that there are qualitatively different kinds of entanglement [6].

We remark that the study of very specific and simply parametrized classes of states, typically satisfying some symmetry (like Werner states [5] or isotropic states [7]), has always turned out to be very useful to improve our understanding of the entanglement phenomenon and of the geometry and properties of the set of states. In this paper we provide examples of states that, despite a simple structure, exhibit interesting properties both in the bipartite and multipartite setting.

One of the means to investigate the entanglement of states is based on the use of linear maps [8, 9] which are positive $(\mathrm{P})$ but not completely positive $(\mathrm{CP})$ : we shall refer to them as PnCP maps. A map is $\mathrm{P}$ if it transforms any state into another positive operator. It is moreover $\mathrm{CP}$ if also its partial action on a subsystem of any larger system gives rise to a $\mathrm{P}$ map. In the case of a bipartite

*Electronic address: piani@ts.infn.it

†Electronic address: caterina.mora@uibk.ac.at system, a state is entangled if and only if there exists a PnCP map such that the operator obtained acting with the map on only one of the two subsystems is not positive any more. The simplest example of PnCP map is the operation of transposition $T$ (with respect to a given basis). The action of transposition on one of the subsystems is called partial transposition (PT) and is also known as the Peres-Horodecki criterion [8, 9]. In the bipartite $2 \otimes 2$ and $2 \otimes 3$ dimensional cases, PT can "detect" all entangled states: only states that develop negative eigenvalues under PT (NPT states) are entangled. In higher dimensions there are states which are positive under partial transposition (PPT) even if entangled. The latter states have the interesting property that their entanglement cannot be distilled (see [2] for a review), thus it is considered to be "bound".

Beside partial transposition, there is another easilycomputable entanglement criterion, realignment [10, 11]. Both PT and realignment are part of the larger family of permutational criteria [12, 13], and they constitute the only two independent criteria of such type in the bipartite scenario. It must be remarked that realignment (i) is not related to a positive linear map and (ii) can detect some PPT bound entangled states.

If we want to use linear PnCP maps to detect PPT bound entangled states, it is necessary to use PnCP maps that are not decomposable, i.e. that cannot be written as the sum of a CP map and a CP map composed with transposition. Indeed, the study of $\mathrm{P}$ maps is strictly related to the study of entanglement, the link being provided by the Choi-Jamiołkowsky isomorphism [14, 15].

It was proved that every entangled state is useful for tasks that it would be impossible to perform classically [16]; in this sense bound entanglement can be "activated" [17]. Quite interestingly, it was found that PPT 
bound entangled states provide probabilistic interconvertibility among multipartite pure states, which are not interconvertible by Local Operations and Classical Communication (LOCC) alone [18, 19].

The first explicit examples of a PPT entangled states were given in [20], and, since then, many other examples have been found [21, 22, 23, 24, 25]. It has been shown [26] that most of these states are part of a same family of PPT - but not a priori entangled - states. A first systematic method to construct PPT bound entangled states was proposed in [27], and is based on the concept of unextendible product basis.

In the present work we first consider a class of bipartite $d \otimes d$ states, with $d$ a non-prime dimension, which are constructed/described by a given set of pure states and a mixing parameter (a probability). Given two states $\psi^{(1)}$ and $\psi^{(2)}$, in a $d_{1} \otimes d_{1}$ and $d_{2} \otimes d_{2}$ Hilbert space, respectively, we consider the set of mixed states parametrized by the mixing parameter $p$

$$
\begin{aligned}
& \rho_{p}\left(\psi^{(1)}, \psi^{(2)}\right)= \\
& \frac{1-p}{\left(d_{1}^{2}-1\right)\left(d_{2}^{2}-1\right)}\left(\mathbb{1}-P_{\psi^{(1)}}\right) \otimes\left(\mathbb{1}-P_{\psi^{(2)}}\right) \\
& +p P_{\psi^{(1)}} \otimes P_{\psi^{(2)}},
\end{aligned}
$$

where $P_{\psi^{(i)}}$ is the projector onto state $\psi^{(i)}$. We will see that, for almost any choice of $\psi^{(1)}$ and $\psi^{(2)}$ entangled, there exists a $p_{\Gamma}>0$ such that $\rho_{p}$ is PPT entangled for all choices of $p<p_{\Gamma}$. The structure of these states can be considered very simple in comparison to the PPT bound entangled states already known in literature. The class is a generalization of states already appeared in [18, 19], where it was proved that some states in the class, even though PPT, are entangled, because they allow operations that are impossible by LOCC. In our case, we prove that they are entangled by constructing canonically associated entanglement witnesses. Moreover, we show that, for almost all choices of the involved pure states and for a suitable range of the mixing parameter, the states are entangled but detected by neither partial transposition nor, in the bipartite case, realignment. This class of states is naturally rich. Furthermore, it can be verified by direct inspection that it is not contained in the class described in [26], thus is contributes effectively to the variety of the known PPT (entangled) states.

We also study the multipartite setting, to which the family of states can be naturally extended [18, 19]. It is possible to show [18] that these states can be PPT entangled with respect to every bipartite cut. As in the bipartite case, this is proved by associating to each state a canonical witness. Furthermore, we find conditions for which the states contain genuine multipartite entanglement, and show that it is possible to have a genuinely multipartite entangled state which is PPT with respect to any bipartite cut. Finally, we relate the properties of $k$-positivity and nondecomposability of linear maps (to be defined in the following), and show that even a decomposable map can become useful to detect PPT entangled states just by considering its trivial extensions.

The paper is organized as follows. In Section II we provide definitions and basic notions. In Section III we introduce the basic set of states of interest, involving the choice of two pure states, and in Section IV we associate to them canonical witnesses. In Section V we discuss partial transposition and realignment and in Section VI we generalize the construction of the class of states relating it to the choice of a set of $M \geq 2$ pure states. Section VII is devoted to some considerations regarding the construction of the canonical witnesses. In Section VII the multipartite setting is studied. In Section IX, starting from considering tensor-like witnesses, we provide a general theorem relating the properties of $k$-positivity and nondecomposability.

\section{DEFINITIONS AND BASIC NOTIONS}

A $d$-dimensional system is associated to the Hilbert space $\mathbb{C}^{d}$, and operators on such system are described by the algebra of $d \times d$ matrices with complex entries $M_{d}$. A state $\rho$ corresponds to a normalized $(\operatorname{Tr}(\rho)=1)$ positive semidefinite $(\rho \geq 0)$ matrix. We will denote (normalized) vectors in the Hilbert space by $|\psi\rangle$ or $\psi$, and the projector onto the pure state $\psi$ by $P_{\psi}=|\psi\rangle\langle\psi|$.

\section{A. Entanglement and separable states}

A bipartite system $\mathrm{AB}$ is associated to a tensorproduct Hilbert space $\mathcal{H}_{A B}=\mathcal{H}_{A} \otimes \mathcal{H}_{B}$. A pure bipartite state $\psi_{A B}$ is entangled if it is not factorized, i.e. not of the form $\psi_{A B}=\psi_{A} \otimes \psi_{B}$. A bipartite mixed state $\rho_{A B}$ is separable if it can be written as a convex combination of factorized states

$$
\rho_{A B}=\sum_{i} p_{i} P_{\psi_{A}^{i}} \otimes P_{\psi_{B}^{i}}, \quad p_{i} \geq 0, \quad \sum_{i} p_{i}=1,
$$

otherwise it is entangled.

More in general one can consider $N$-partite systems, that are associated to tensor-product Hilbert spaces of the form $\bigotimes_{i=1}^{N} \mathcal{H}_{i}$, where $\mathcal{H}_{i}$ is the Hilbert space associated to system $i$. In this case it is possible to study the separability issue with respect to different groupings of the parties. A pure $N$-partite state $\psi_{N}$ is $k$-separable if it can be written as a tensor product of $k$ states, i.e. as $\psi_{N}=\bigotimes_{i=1}^{k} \psi_{S_{i}}$, with $\mathcal{P}_{k}=\left\{S_{i}\right\}_{i=1}^{k}$ a partition of the parties in $k$ subsets. In particular, $\psi_{N}$ is biseparable if $\psi_{N}=\psi_{S_{1}} \otimes \psi_{S_{2}}$. A pure state is $k$-partite entangled if it cannot be written as the tensor product of states, each of which pertains to less than $k$ parties. Similarly, a mixed state is $k$-separable if it can be written as a convex combination of $k$-separable pure states. The $k$-partition need not be the same for all the $k$-separable pure states entering in the convex combination; if all the pure states can be chosen to be $k$-separable with respect to the same 
partition $\mathcal{P}_{k}$, we say that the state is $k$-separable with respect to the partition $\mathcal{P}_{k}$. In particular, we say that a state is biseparable if it is 2-separable, and that it is separable along a cut $S_{1}: S_{2}$ if it is 2-separable with respect to the partition $\left\{S_{1}, S_{2}\right\}$. A mixed state is $k$-partite entangled if every possible convex decomposition of the state contains at least a $k$-partite entangled pure state. Notice that a $N$-partite state is biseparable if and only if it is not $N$-partite entangled. Any result valid in the bipartite setting can be applied to the multipartite case when considering a given cut.

In the bipartite case, any pure state $\psi$ can be written in its standard Schmidt decomposition:

$$
|\psi\rangle=\sum_{i=1}^{r} \mu_{i}\left|i_{A} \otimes i_{B}\right\rangle,
$$

where $\mu_{i}>0, \sum_{i=1}^{r} \mu_{i}^{2}=1$, are the Schmidt coefficients, $r \leq \min \left(d_{A}, d_{B}\right)$ is the Schmidt rank (or number) and $\left|i_{A(B)}\right\rangle$ are orthogonal states (i.e. they can be extended to an orthonormal basis). We say that a bipartite density matrix $\sigma$ has Schmidt number $k$ if (i) for any decomposition $\left\{p_{i} \geq 0, \phi_{i}\right\}$ of $\sigma$, i.e. $\sigma=\sum_{i} p_{i}\left|\phi_{i}\right\rangle\left\langle\phi_{i}\right|$, at least one of the vectors $\phi_{i}$ has at least Schmidt rank $k$ and (ii) there exists a decomposition of $\sigma$ with all vectors $\left\{\phi_{i}\right\}$ of Schmidt rank at most $k$ [28].

\section{B. Partial transposition and realignment}

We recall now the two separability criteria which we will use in the following and which are based on the reordering of the entries of the density matrix: partial transposition [8, 9] and realignment [10, 11]. Given a bipartite density matrix $\rho=\sum_{i j k l} \rho_{i j, k l}|i j\rangle\langle k l|$ the linear operations of partial transposition and realignment are defined as follows. Partial transposition (with respect to the first system) corresponds to the reordering $(|i j\rangle\langle k l|)^{\Gamma_{A}}=|k j\rangle\langle i l|$, and realignment to $R(|i j\rangle\langle k l|)=$ $|i k\rangle\langle j l|$. It is immediate to see that, if a state is separable, then both $\left\|\rho_{A B}^{\Gamma_{A}}\right\|_{1} \leq 1$ and $\left\|R\left(\rho_{A B}\right)\right\|_{1} \leq 1$ must hold, with $\|X\|_{1}=\operatorname{Tr} \sqrt{X^{\dagger} X}$ the trace norm of $X$. The condition $\left\|\rho_{A B}^{\Gamma_{A}}\right\|_{1} \leq 1$ is equivalent to requiring that $\rho_{A B}$ stays positive under partial transposition, i.e. $\rho_{A B}^{\Gamma_{A}} \geq 0$.

As regards partial transposition, we note that for any bipartite state $|\psi\rangle=\sum_{j} \mu_{j}|i i\rangle$ (here written in its Schmidt decomposition), we have

$$
\begin{aligned}
(|\psi\rangle\langle\psi|)^{\Gamma}=\sum_{j} & \mu_{j}^{2}|j j\rangle\langle j j| \\
& +\sum_{j>i} \mu_{i} \mu_{j}\left(\left|\psi_{i j}^{+}\right\rangle\left\langle\psi_{i j}^{+}|-| \psi_{i j}^{-}\right\rangle\left\langle\psi_{i j}^{-}\right|\right),
\end{aligned}
$$

with $\left|\psi_{i j}^{ \pm}\right\rangle=(|i j\rangle \pm|j i\rangle) / \sqrt{2}$, and where partial transposition was operated in the Schmidt basis. The eigenvalues of $(|\psi\rangle\langle\psi|)^{\Gamma}$ are $\lambda_{0}^{i}=\mu_{i}^{2}$, for $i=1, \ldots, d$, and $\lambda_{ \pm}^{i j}= \pm \mu_{i} \mu_{j}$, for $j>i$, corresponding to Schmidtrank-one eigenstates $|i i\rangle$ and Schmidt-rank-two eigenstates $\left|\psi_{i j}^{ \pm}\right\rangle$, respectively. Thus, either $\psi$ is factorized, i.e. there is only one non-vanishing Schmidt coefficient (equal to 1), or all the eigenvalues of $(|\psi\rangle\langle\psi|)^{\Gamma}$ have modulus strictly less than one. As regards realignment, we have $R(|\psi\rangle\langle\psi|)=\sum_{i j} \mu_{i} \mu_{j}|i\rangle\langle i|\otimes| j\rangle\langle j|$. For any pure state $\psi$, thus, $\left\|(|\psi\rangle\langle\psi|)^{\Gamma_{A}}\right\|_{1}=\|R(|\psi\rangle\langle\psi|)\|_{1}=\left(\sum_{i} \mu_{i}\right)^{2}$. Therefore, both partial transposition and realignment detect all pure entangled (bipartite) states.

\section{Entanglement witnesses}

It is well known that any bipartite entangled state $\rho_{A B}$ can be detected by means of a suitable entanglement witness [9, 29]: for every entangled state $\rho_{A B}$ there exists an observable $W=W_{A B}$ such that $\operatorname{Tr}\left(W \rho_{A B}\right)<0$, while $\operatorname{Tr}\left(W \sigma_{\text {sep }}\right) \geq 0$ for all separable states $\sigma_{\text {sep. }}$. It is clear that a nontrivial entanglement witness, i.e. an observable able to detect at least some entangled state, is not positive semidefinite.

A witness is decomposable 30] if it can be written as $W=P+Q^{\Gamma}$, with $P, Q \geq 0$ positive semidefinite operators. To detect PPT entangled states a witness must be nondecomposable. Indeed, $\operatorname{Tr}(W \rho) \geq 0$ for all PPT state $\rho$ and all decomposable witnesses $W$.

In [28, 31] the concept of Schmidt-number witness was introduced. A (nontrivial) Schmidt-number $k$ witness $W$ is an observable which is positive semidefinite with respect to (mixed) states of Schmidt number $k-1$, but such that there exists a Schmidt-number $k$ state $\rho$ such that $\operatorname{Tr}(W \rho)<0$. Notice that standard entanglement witnesses correspond to Schmidt-number 2 witnesses.

Moreover, witnesses are able to distinguish between different kinds of multipartite entanglement [32]. Indeed, there always exists an observable whose expectation value is able to discriminate between states in a convex subset and a state outside it. Therefore, for any state $\rho$ that is $(k+1)$-partite entangled there exists a witness $W$ such that $\operatorname{Tr}(W \rho)<0$ while $\operatorname{Tr}(W \sigma) \geq 0$ for all states $\sigma$ that are at most $k$-partite entangled. Similarly, there is always a witness which distinguishes a state that is not $k$-separable from states that are so. In particular, for an $N$-partite state that is $N$-partite entangled, there exists a witness which tells it from biseparable states.

\section{Maps and entanglement}

A linear map $\Lambda: M_{d} \rightarrow M_{d^{\prime}}$ is: positive if $\Lambda[X] \geq 0$ for all $X \geq 0 ; k$-positive if $\operatorname{id}_{k} \otimes \Lambda$ is positive, with $\mathrm{id}_{k}$ the identity map on $M_{k}$; completely positive if it is $k$-positive for all $k \geq 1$. It is remarkable that $\Lambda: M_{d} \rightarrow M_{d^{\prime}}$ is completely positive if and only if it is $d$-positive [14].

Operators $W$ in $M_{d d^{\prime}} \cong M_{d} \otimes M_{d^{\prime}}$ are isomorphic to linear maps $\Lambda: M_{d} \rightarrow M_{d^{\prime}}$, through the Choi- 
Jamiołkowski isomorphism [14, 15]:

$$
\begin{aligned}
W & =W_{\Lambda}=d\left(\operatorname{id}_{d} \otimes \Lambda\right)\left[P_{d}^{+}\right] \\
\Lambda[X] & =\Lambda_{W}[X]=\operatorname{Tr}_{1}\left(\left(X^{T} \otimes \mathbb{1}\right) W\right),
\end{aligned}
$$

where the trace in (5) is on the the first subsystem only, and

$$
P_{d}^{+} \equiv P_{\Psi_{d}^{+}}, \quad\left|\Psi_{d}^{+}\right\rangle=\frac{1}{\sqrt{d}} \sum_{i}|i \otimes i\rangle
$$

is the maximally entangled state for a $d \otimes d$ system, $d \geq 2$.

In particular, (nontrivial) witnesses are isomorphic to PnCP maps. An example of PnCP map is transposition, that fails already to be 2-positive, and is associated to $V=d\left(\operatorname{id}_{d} \otimes T\right)\left[P_{d}^{+}\right]$, that is the swap operator: $V \mid \phi \otimes$ $\chi\rangle=|\chi \otimes \phi\rangle$. In the same way as there is always an entanglement witness which detects a bipartite entangled states $\rho_{A B}$, there is also a $\mathrm{PnCP}$ map $\Lambda$ such that

$$
\left(\operatorname{id}_{A} \otimes \Lambda_{B}\right)\left[\rho_{A B}\right] \geq 0
$$

is not satisfied [9].

Every nondecomposable witness is associated to a nondecomposable map [30]. A map $\Lambda$ is decomposable if it can be written as $\Lambda=\Lambda_{1}^{\mathrm{CP}}+\Lambda_{2}^{\mathrm{CP}} \circ T$, where $\Lambda_{1(2)}^{\mathrm{CP}}$ is a completely positive map and $\circ$ stands for composition. Indeed, Eq. (7) is satisfied for all PPT states and decomposable maps. Moreover, every (non-trivial) Schmidtnumber $k$ witness is associated to a $(k-1)$-positive but not $k$-positive map [28, 31].

\section{THE BASIC SET OF STATES}

We start by considering a bipartite system with associated Hilbert space $\mathcal{H}_{A B}=\mathcal{H}_{A} \otimes \mathcal{H}_{B}$, with

$$
\mathcal{H}_{A}=\mathcal{H}_{A_{1}} \otimes \mathcal{H}_{A_{2}}, \quad \mathcal{H}_{B}=\mathcal{H}_{B_{1}} \otimes \mathcal{H}_{B_{2}}
$$

and $\mathcal{H}_{A_{i}}=\mathcal{H}_{B_{i}}=\mathbb{C}^{d_{i}}$.

We focus on states

$$
\rho_{A B}\left(\psi^{(1)}, \psi^{(2)}\right)=\rho_{1}\left(\psi^{(1)}\right) \otimes \rho_{2}\left(\psi^{(2)}\right),
$$

with

$$
\rho_{i}\left(\psi^{(i)}\right)=\rho_{A_{i} B_{i}}=\mathcal{N}_{i}\left(\mathbb{1}-P_{\psi^{(i)}}\right)_{A_{i} B_{i}},
$$

where $\mathcal{N}_{i}=1 /\left(d_{i}^{2}-1\right)$ are normalization factors. Each pure state $\left|\psi^{(i)}\right\rangle \equiv\left|\psi^{(i)}\right\rangle_{A_{i} B_{i}}$ is given by

$$
\left|\psi^{(i)}\right\rangle=\sum_{j=1}^{r_{i}} \mu_{j}^{(i)}\left|j_{A_{i}} \otimes j_{B_{i}}\right\rangle
$$

where (11) and $r_{i}$ are the corresponding Schmidt decomposition and Schmidt number, respectively. The states $\rho_{i}\left(\psi^{(i)}\right)$ are $A_{i}: B_{i}$ separable [33], thus $\rho_{A B}\left(\psi^{(1)}, \psi^{(2)}\right)$ is $A: B$ separable. The partial transposition of $\rho_{i}\left(\psi^{(i)}\right)$ with respect to $A_{i}$ is:

$$
\rho_{i}^{\Gamma_{A}}\left(\psi^{(i)}\right)=\mathcal{N}_{i}\left(\mathbb{1}-\left(\left|\psi^{(i)}\right\rangle\left\langle\psi^{(i)}\right|\right)^{\Gamma_{A}}\right) .
$$

From what we have seen about partial transposition of pure states in Section III, it is clear that $\rho_{i}^{\Gamma_{A}}\left(\psi^{(i)}\right)$ has full rank if and only if $\psi^{(i)}$ is entangled. It follows that, if both $\psi^{(1)}$ and $\psi^{(2)}$ are entangled, $\rho_{A B}^{\Gamma_{A}}\left(\psi^{(1)}, \psi^{(2)}\right)$ is strictly positive. This implies that a change of $\rho_{A B}\left(\psi^{(1)}, \psi^{(2)}\right)$ small enough cannot spoil the positivity of the partial transpose.

This leads us to define the class of states of interest consisting of the convex combination (11). Such class has already appeared in literature [18, 19], and it was proved that some states in the class are entangled even if PPT, by showing that they allow to perform tasks that are impossible under LOCC. Here, in a different vein, we will look for entanglement witnesses to prove that, for almost all choices of entangled states $\psi^{(1)}$ and $\psi^{(2)}$, the state $\rho_{p}\left(\psi^{(1)}, \psi^{(2)}\right)$ is entangled as soon as $p>0$. Therefore, if $p$ is chosen to be small enough, $\rho_{p}\left(\psi^{(1)}, \psi^{(2)}\right)$ is a PPT entangled state. We remark that if one of the two states $\psi^{(i)}$ is separable, while the other is entangled, then $\rho_{p}\left(\psi^{(1)}, \psi^{(2)}\right)$ is always NPT; $\rho_{p}\left(\psi^{(1)}, \psi^{(2)}\right)$ can be made PPT entangled, for some choice of $p>0$, only if both pure states $\psi^{(i)}$ are entangled.

\section{CANONICAL WITNESS}

We now construct a suitable entanglement witness. If we complete $\psi^{(i)}$ to a basis $\left\{\psi_{1}^{(i)} \equiv \psi^{(i)}, \psi_{2}^{(i)}, \ldots, \psi_{d_{i}^{2}}^{(i)}\right\}$ of $\mathbb{C}^{d_{i}^{2}}$, for both $i=1,2$, we see that $\rho_{p}\left(\psi^{(1)}, \psi^{(2)}\right)$ is diagonal in the basis $\left\{\psi_{i}^{(1)} \otimes \psi_{j}^{(2)}\right\}$ :

$$
\rho_{p}\left(\psi^{(1)}, \psi^{(2)}\right)=\sum_{i=1}^{d_{1}^{2}} \sum_{j=1}^{d_{2}^{2}} p_{i j} P_{\psi_{i}^{(1)}} \otimes P_{\psi_{j}^{(2)}}
$$

with $p_{i j} \geq 0$. Note that, since the tensor product structure is along the $A_{1} B_{1}: A_{2} B_{2}$ cut, and not along the $A_{1} A_{2}: B_{1} B_{2}$ cut, the expression (13) is not related to the $A: B$ separability property given by (2). We will consider witnesses diagonal in the same basis, i.e.

$$
W=\sum_{i=1}^{d_{1}^{2}} \sum_{j=1}^{d_{2}^{2}} w_{i j} P_{\psi_{i}^{(1)}} \otimes P_{\psi_{j}^{(2)}},
$$

so that $\operatorname{Tr}\left(W \rho_{p}\left(\psi^{(1)}, \psi^{(2)}\right)\right)=\sum_{i j} p_{i j} w_{i j}$. Of course, the operator (14) is not a trivial witness only if $w_{i j}<0$ for some $(i, j)$. In particular, if we define

$$
\begin{aligned}
W_{\epsilon}\left(\psi^{(1)}, \psi^{(2)}\right) & =P_{\psi^{(1)}} \otimes\left(\mathbb{1}-P_{\psi^{(2)}}\right) \\
& +\left(\mathbb{1}-P_{\psi^{(1)}}\right) \otimes P_{\psi^{(2)}} \\
& -\epsilon P_{\psi^{(1)}} \otimes P_{\psi^{(2)}} \\
& =P_{\psi^{(1)}} \otimes \mathbb{1}+\mathbb{1} \otimes P_{\psi^{(2)}} \\
& -(2+\epsilon) P_{\psi^{(1)}} \otimes P_{\psi^{(2)}},
\end{aligned}
$$




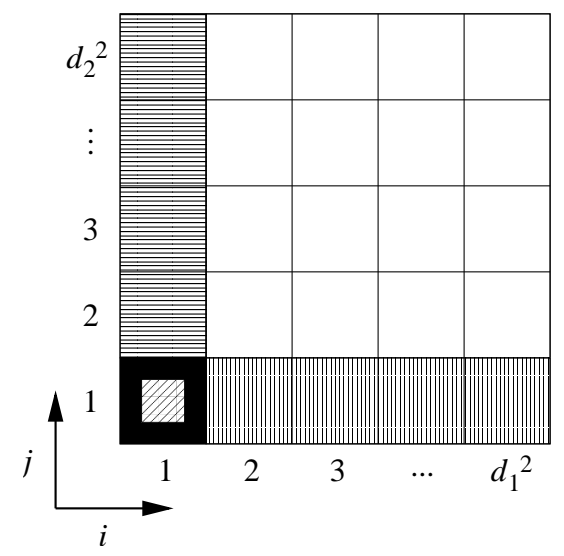

FIG. 1: Graphical representation of the choice of $p_{i j}$ (white and black) and $w_{i j}$ (patterns) in (1) and (15), respectively. White color corresponds to the separable part of $\rho_{p}\left(\psi^{(1)}, \psi^{(2)}\right)$, while the vertical and horizontal patterns correspond to the positive part of the witness $W_{\epsilon}\left(\psi^{(1)}, \psi^{(2)}\right)$. Black color and the diagonal pattern stand for $p_{11}=p>0$ and $w_{11}=-\epsilon<0$, respectively (see the main text for details).

with $\epsilon \geq 0$, we have

$$
\operatorname{Tr}\left(W_{\epsilon}\left(\psi^{(1)}, \psi^{(2)}\right) \rho_{p}\left(\psi^{(1)}, \psi^{(2)}\right)\right)=-p \epsilon .
$$

Indeed, $\rho_{p}\left(\psi^{(1)}, \psi^{(2)}\right)$ and $W_{\epsilon}\left(\psi^{(1)}, \psi^{(2)}\right)$ are diagonal in the same basis and their supports are orthogonal except for the unidimensional subspace spanned by $\psi^{(1)} \otimes \psi^{(2)}$. A graphical representation of both the state and the witness decompositions (in terms of $p_{i j}$ and $w_{i j}$ ) for the choices (11) and (15) is given in Fig. 11.

We have to prove that, at least for some choices of $\psi^{(i)}$, there exists $\epsilon>0$ such that $W_{\epsilon}\left(\psi^{(1)}, \psi^{(2)}\right)$ is a nontrivial entanglement witness. Indeed, as soon as $\epsilon>0$, $W_{\epsilon}\left(\psi^{(1)}, \psi^{(2)}\right)$ is not a positive semidefinite operator. We proceed by finding the conditions for which it is positive on separable states: $\left\langle\alpha_{A} \otimes \beta_{B}\left|W_{\epsilon}\left(\psi^{(1)}, \psi^{(2)}\right)\right| \alpha_{A} \otimes \beta_{B}\right\rangle \geq$ 0 , for all factorized (not necessarily normalized) $\mid \alpha_{A} \otimes$ $\left.\beta_{B}\right\rangle$. Let us therefore consider vectors

$$
\begin{aligned}
& |\alpha\rangle=\sum_{i=1}^{d_{1}} \sum_{j=1}^{d_{2}} \alpha_{i j}\left|i_{A_{1}} \otimes j_{A_{2}}\right\rangle \\
& |\beta\rangle=\sum_{i=1}^{d_{1}} \sum_{j=1}^{d_{2}} \beta_{i j}\left|i_{B_{1}} \otimes j_{B_{2}}\right\rangle,
\end{aligned}
$$

where $\alpha=\left[\alpha_{i j}\right], \beta=\left[\beta_{i j}\right]$ are complex $d_{1} \times d_{2}$ rectangular matrices, and where we have taken the bases $\left\{\left|i_{A_{k}}\right\rangle\right\}$, $\left\{\left|i_{B_{k}}\right\rangle\right\}$ in the Hilbert spaces $\mathcal{H}_{A_{k}}, \mathcal{H}_{B_{k}}, k=1,2$ to be the ones corresponding to the Schmidt decomposition (11) of $\psi^{(1)}$ and $\psi^{(2)}$. We find

$$
\begin{gathered}
\left\langle\alpha_{A} \otimes \beta_{B}\left|W_{\epsilon}\left(\psi^{(1)}, \psi^{(2)}\right)\right| \alpha_{A} \otimes \beta_{B}\right\rangle= \\
\operatorname{Tr}\left(\left(\beta^{T} \mu^{(1)} \alpha\right)^{\dagger}\left(\beta^{T} \mu^{(1)} \alpha\right)\right)+\operatorname{Tr}\left(\left(\alpha \mu^{(2)} \beta^{T}\right)^{\dagger}\left(\alpha \mu^{(2)} \beta^{T}\right)\right) \\
-(2+\epsilon) \mid \operatorname{Tr}\left(\left.\alpha \mu^{(2)} \beta^{T} \mu^{(1)}\right|^{2}, \quad\right. \text { (17) }
\end{gathered}
$$

with $\mu^{(i)}=\left(\mu^{(i)}\right)^{\dagger}=\left(\mu^{(i)}\right)^{T}$ the positive diagonal matrix of the Schmidt coefficients of $\psi^{(i)}$.

Let us consider a matrix orthonormal basis (o.n.b.) in $M_{d}$, i.e. a set of matrices $\left\{F_{i}\right\}_{i=1}^{d^{2}}$ such that the matrices are orthonormal with respect to the Hilbert-Schmidt inner product: $\operatorname{Tr}\left(F_{i}^{\dagger} F_{j}\right)=\delta_{i j}$. For any matrix o.n.b. $\left\{F_{i}\right\}$ and any matrix $X$, we have $X=\sum_{i} \operatorname{Tr}\left(F_{i}^{\dagger} X\right) F_{i}$, and $\sum_{i}\left|\operatorname{Tr}\left(F_{i}^{\dagger} X\right)\right|^{2}=\operatorname{Tr}\left(X^{\dagger} X\right)$. As $\operatorname{Tr}\left(\mu^{(i)^{2}}\right)=1$, each $\mu^{(i)}$ can be be considered as an element of a matrix o.n.b in $M_{d_{i}}$. Let us complete each $G_{1}^{(i)}=\mu^{(i)}$ to an o.n.b. $\left\{G_{j}^{(i)}\right\}_{j=1}^{d_{i}^{2}}$. Then

$$
\begin{aligned}
\left|\operatorname{Tr}\left(\alpha \mu^{(2)} \beta^{T} \mu^{(1)}\right)\right|^{2} & \leq \sum_{j=1}^{d_{1}^{2}}\left|\operatorname{Tr}\left(\alpha \mu^{(2)} \beta^{T} G_{j}^{(1)}\right)\right|^{2} \\
& =\operatorname{Tr}\left(\left(\alpha \mu^{(2)} \beta^{T}\right)^{\dagger}\left(\alpha \mu^{(2)} \beta^{T}\right)\right)
\end{aligned}
$$

and, similarly,

$$
\begin{aligned}
\left|\operatorname{Tr}\left(\beta^{T} \mu^{(1)} \alpha \mu^{(2)}\right)\right|^{2} & \leq \sum_{j=1}^{d_{2}^{2}}\left|\operatorname{Tr}\left(\beta^{T} \mu^{(1)} \alpha G_{j}^{(2)}\right)\right|^{2} \\
& =\operatorname{Tr}\left(\left(\beta^{T} \mu^{(1)} \alpha\right)^{\dagger}\left(\beta^{T} \mu^{(1)} \alpha\right)\right) .
\end{aligned}
$$

Inequalities (18) correspond to $P_{\psi^{(1)}} \otimes P_{\psi^{(2)}} \leq \mathbb{1} \otimes P_{\psi^{(2)}}$ and $P_{\psi^{(1)}} \otimes P_{\psi^{(2)}} \leq P_{\psi^{(1)}} \otimes \mathbb{1}$, respectively. Yet having cast them in the form (18) allows us to argue about the necessary and sufficient conditions on $\psi^{(1)}$ and $\psi^{(2)}$ to have a non-trivial witness $W_{\epsilon}\left(\psi^{(1)}, \psi^{(2)}\right)$, i.e. to have $\epsilon>0$.

Positivity on factorized states imposes $\epsilon=0$ if and only if there are matrices $\alpha$ and $\beta$ such that the inequalities (18) are both saturated at the same time, i.e. both sums in (18) reduce to just the first term, and this term does not vanish. Indeed, under these conditions, (17) is equal to $-\epsilon \mid \operatorname{Tr}\left(\left.\alpha \mu^{(2)} \beta^{T} \mu^{(1)}\right|^{2}\right.$ and strictly negative as soon as $\epsilon>0$. The two sums reduce to the first term if and only if there are $\alpha$ and $\beta$ such that 34.

$$
\alpha \mu^{(2)} \beta^{T}=\mu^{(1)}=G_{1}^{(1)}, \quad \beta^{T} \mu^{(1)} \alpha=c \mu^{(2)}=c G_{1}^{(2)}
$$

where $c$ is a complex constant of proportionality. Only in this case, in fact, $\alpha \mu^{(2)} \beta^{T}$ and $\beta^{T} \mu^{(1)} \alpha$ are orthogonal to all the other elements of the two matrix o.n.b.. If condition (19) is satisfied, the first two terms on the right side of (17) must be equal. Thus one finds $|c|=1$ and finally, taking into account Hermiticity and positivity of $\mu^{(i)}$, one obtains $c=1$.

We have reduced the problem of determining the existence of a non-trivial witness of the form (15), to that of verifying whether, for given states $\psi^{(1)}$ and $\psi^{(2)}$, there exist matrices $\alpha$ and $\beta$ which solve the system of matrix 
equations

$$
\begin{aligned}
& \alpha \mu^{(2)} \beta^{T}=\mu^{(1)} \\
& \beta^{T} \mu^{(1)} \alpha=\mu^{(2)} .
\end{aligned}
$$

First, we notice that this is possible only if $\mu^{(2)}$ and $\mu^{(1)}$ have the same rank, i.e. only if the states $\psi^{(1)}$ and $\psi^{(2)}$ have the same Schmidt number $r=r_{1}=r_{2}$. It is sufficient to focus on this case. We further observe that w.l.o.g. we can consider the $r$ non-vanishing Schmidt coefficients of $\psi^{(i)}$ to appear in the first $r$ diagonal entries of $\mu^{(i)}$, for $i=1,2$. Therefore, we can consider all the matrices entering (20) to be $r \times r$ square matrices, even if the initial dimensions $d_{1}$ and $d_{2}$ were different. Moreover, they are all invertible, since we are considering the case the rank of both Schmidt coefficient matrices $\mu^{(i)}$ is $r$. We can therefore rewrite (20) as $\mu^{(2)} \beta^{T}=\alpha^{-1} \mu^{(1)}$ and $\beta^{T} \mu^{(1)}=\mu^{(2)} \alpha^{-1}$. Taking into account that both matrices $\mu^{(i)}$ are diagonal and strictly positive (i.e. all the $r$ Schmidt coefficients are not null), we arrive at the following relations:

$$
\begin{aligned}
\left(\alpha^{-1}\right)_{i j} & =\frac{\mu_{i}^{(2)}}{\mu_{j}^{(1)}} \beta_{j i}, \\
\beta_{j i}\left(\mu_{j}^{(1)}\right)^{2} & =\beta_{j i}\left(\mu_{i}^{(2)}\right)^{2} .
\end{aligned}
$$

From (21a), we have that if $\beta_{j i}=0$ then also $\left(\alpha^{-1}\right)_{i j}=0$; from (21b) we find that, if $\beta_{j i} \neq 0$, then $\mu_{j}^{(1)}=\mu_{i}^{(2)}$ and therefore, from (21a), $\left(\alpha^{-1}\right)_{i j}=\beta_{j i}$. In conclusion, we have $\alpha^{-1}=\beta^{T}$. Therefore, a solution to equations (20) exists only if $\mu^{(1)}$ and $\mu^{(2)}$ are connected by a similarity transformation

$$
\alpha \mu^{(2)} \alpha^{-1}=\mu^{(1)},
$$

and have the same eigenvalues. In such case, we have that (17) reduces to $-\epsilon \operatorname{Tr}\left(\left(\mu^{(i)}\right)^{2}\right)=-\epsilon$, so that we must choose $\epsilon=0$ to have positivity on separable states.

We have shown that the witness $W_{\epsilon}\left(\psi^{(1)}, \psi^{(2)}\right)$ defined in (15) can always be chosen to be non-trivial, i.e. with $\epsilon>0$, except in the case $\psi^{(1)}$ and $\psi^{(2)}$ have essentially the same Schmidt decomposition. Notice that w.l.o.g. we can consider the Schmidt coefficients to be ordered as $\mu_{k}^{(i)} \geq \mu_{k+1}^{(i)}$, for $i=1,2$. Thus, we have always a witness except in the case $\mu^{(1)}=\mu^{(2)}$ (indeed, the similarity transformation (22) is actually a permutation). Correspondingly, we have proved that, for almost all pairs of pure entangled states $\psi^{(i)}, i=1,2$, the state $\rho_{p}\left(\psi^{(1)}, \psi^{(2)}\right)$ is entangled as soon as $p>0$.

\section{PARTIAL TRANSPOSITION AND REALIGNMENT}

Let us now consider more in detail the behavior of the class of states $\rho_{p}\left(\psi^{(1)}, \psi^{(2)}\right)$ under the operations of partial transposition and realignment [35]. Partial transposition for such states has already been studied in [18]. For completeness, we reproduce here those results, and we extend the analysis by comparing the entanglement detection power of partial transposition and realignment. Moreover, we observe that no element in the class is a candidate to be an NPT bound state, i.e. as soon as the states are NPT, they are provably distillable.

As regards $\mathrm{PT}$, we have that the eigenvalues of $\rho_{p}^{\Gamma_{A}}\left(\psi^{(1)}, \psi^{(2)}\right)$ are $(1-p) \mathcal{N}_{1} \mathcal{N}_{2}\left(1-\lambda^{(1)}\right)\left(1-\lambda^{(2)}\right)+$ $p \lambda^{(1)} \lambda^{(2)}$, where the $\lambda^{(i)} \mathrm{S}$ run over eigenvalues of $\left(\left|\psi^{(i)}\right\rangle\left\langle\psi^{(i)}\right|\right)^{\Gamma}, \quad i=1,2$. Let us recall that a state $\rho_{A B}$ is distillable if and only if there exist a number of copies $n$ and a Schmidt rank 2 state $\phi_{2}$ such that $\left\langle\phi_{2}\left|\left(\rho^{\Gamma_{A}}\right)^{\otimes n}\right| \phi_{2}\right\rangle<0$ [36]. It is easy to see that the minimum eigenvalue of $\rho_{p}^{\Gamma A}\left(\psi^{(1)}, \psi^{(2)}\right)$ is of the form

$$
(1-p) \mathcal{N}_{1} \mathcal{N}_{2}\left(1-\left(\mu_{k}^{(i)}\right)^{2}\right)\left(1+\mu_{m}^{(j)} \mu_{n}^{(j)}\right)-p\left(\mu_{k}^{(i)}\right)^{2} \mu_{m}^{(j)} \mu_{n}^{(j)},
$$

with $m \neq n$ and $(i, j) \in\{(1,2),(2,1)\}$, i.e. it corresponds to a Schmidt rank 2 eigenvector $\left|k_{A_{i}} k_{B_{i}}\right\rangle \otimes\left|\psi_{m n}^{-}\right\rangle_{A_{j} B_{j}}$. Therefore, as soon as the state is NPT, we prove that it is also distillable by considering $n=1$ and taking as $\phi_{2}$ the eigenvector corresponding to the minimal negative eigenvalue. On the other hand, by choosing $p$ small enough, it always possible to make the smallest eigenvalue positive, if the first term in (23) is not null, i.e. if both states $\psi^{(i)}$ are entangled. More precisely, it can be shown [18] that the necessary and sufficient condition for the state to be $\mathrm{PPT}$ is

$$
\begin{aligned}
\frac{p}{(1-p) \mathcal{N}_{1} \mathcal{N}_{2}} \leq \min \left\{\frac{\left(1-\left(\mu_{1}^{(1)}\right)^{2}\right)\left(1+\mu_{1}^{(2)} \mu_{2}^{(2)}\right)}{\left(\mu_{1}^{(1)}\right)^{2} \mu_{1}^{(2)} \mu_{2}^{(2)}},\right. \\
\left.\frac{\left(1-\left(\mu_{1}^{(2)}\right)^{2}\right)\left(1+\mu_{1}^{(1)} \mu_{2}^{(1)}\right)}{\left(\mu_{1}^{(2)}\right)^{2} \mu_{1}^{(1)} \mu_{2}^{(1)}}\right\} .
\end{aligned}
$$

In particular, to calculate the smallest eigenvalue of the partially-transposed state, it is sufficient to consider only the two biggest Schmidt coefficients of $\psi^{(1)}$ and $\psi^{(2)}$. We will denote by $p_{\Gamma}$ the largest value of $p$ for which $\rho_{p}\left(\psi^{(1)}, \psi^{(2)}\right)$ is PPT. In Fig. 2 we plot the dependence of $p_{\Gamma}$ on the Schmidt coefficients of the two pure states in the case $d_{1}=d_{2}=2$ (i.e. when $\rho_{p}\left(\psi^{(1)}, \psi(2)\right)$ is a state of four qubits).

The condition to determine when the realignment criterion detects entanglement is not trivial to handle analytically. Thus, we will restrict ourselves to the case in which the two pure states $\psi^{(1)}$ and $\psi^{(2)}$ are maximally entangled. In this case, we have

$$
\begin{gathered}
R\left(\rho_{p}\left(\Psi_{d_{1}}^{+}, \Psi_{d_{2}}^{+}\right)\right)=(1-p) \mathcal{N}_{1} \mathcal{N}_{2}\left(\sum_{i=1}^{d_{1}}|i i\rangle\langle j j|-\frac{\mathbb{1}}{d_{1}}\right) \otimes \\
\left(\sum_{i, j=1}^{d_{2}}|i i\rangle\langle j j|-\frac{\mathbb{1}}{d_{2}}\right)+p \frac{\mathbb{1}}{d_{1} d_{2}} .
\end{gathered}
$$




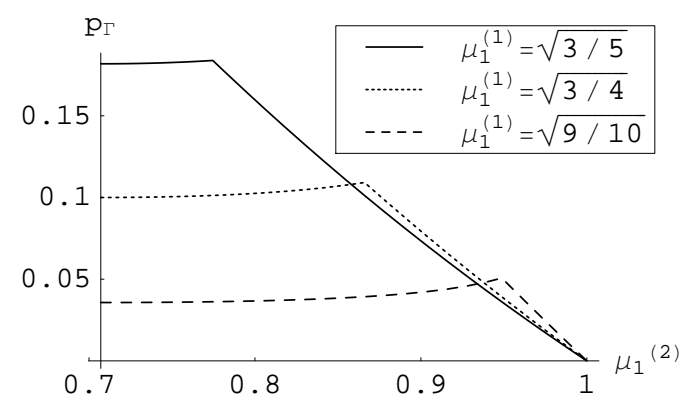

FIG. 2: Dependence of the threshold probability $p_{\Gamma}$ on $\psi^{(2)}$ for fixed choices of $\psi^{(1)}$ in the case $d_{1}=d_{2}=2$. The state $\rho_{p}\left(\psi^{(1)}, \psi^{(2)}\right)$ is entangled for all choices of $\mu_{1}^{(1)} \neq \mu_{1}^{(2)}$; when $0 \leq p<p_{\Gamma}$ the state is PPT, while it is NPT if $p_{\Gamma}<p \leq 1$. The point in which the minimum on the right hand side of (24) changes from one element to the other is clear from the sharp change in the behavior of the curve, and it coincides with the point $\mu_{1}^{(1)}=\mu_{1}^{(2)}$ (note that, for such point, with the methods introduced in this work we are not able to say that the state $\rho_{p}\left(\psi^{(1)}, \psi^{(2)}\right)$ is entangled when PPT (see main text and Table I). As expectable, the threshold value of $p_{\Gamma}$ goes to 0 as one of the two states becomes less entangled.

The condition $\left\|R\left(\rho_{p}\left(\Psi_{d_{1}}^{+}, \Psi_{d_{2}}^{+}\right)\right)\right\|>1$ is thus satisfied only for $p>\frac{d_{1} d_{2}-2}{d_{1}^{2}\left(d_{2}^{2}-2\right)}$, where we have assumed w.l.o.g. $d_{2} \geq d_{1}$. Note that this value is always greater than $p_{\Gamma}=$ $\frac{1}{1+\left(d_{1}+1\right)\left(d_{2}-1\right)}$ : thus, in the case in which the pure states $\psi^{(1)}$ and $\psi^{(2)}$ are maximally entangled, realignment is always less sensitive than PT.

In Section VI we will provide analytical examples of states, which have a structure similar (see Eq. (26)) to that of $\rho_{p}\left(\psi^{(1)}, \psi^{(2)}\right)$, detected as entangled by realignment but not by partial transposition. In Fig. 3 we show that realignment and partial transposition are inequivalent (i.e., there are entangled states detected by one criterion but not by the other one) also in the class $\rho_{p}\left(\psi^{(1)}, \psi^{(2)}\right)$. The plot of Fig. [3] is relevant also for another reason: it shows that also states for which it is not possible to construct a non-trivial witness $W_{\epsilon}\left(\psi^{(1)}, \psi^{(2)}\right)$ (i.e., states for which $\mu^{(1)}=\mu^{(2)}$ ) may be entangled.

For the sake of clarity, in Table I we summarize the relation between the entanglement properties of the two pure states $\psi^{(1)}, \psi^{(2)}$, and those of $\rho_{p}\left(\psi^{(1)}, \psi^{(2)}\right)$.

\section{GENERALIZATION TO MORE THAN TWO STATES $\psi^{(i)}$}

It is possible to straightforwardly generalize the construction of the states $\rho_{p}\left(\psi^{(1)}, \psi^{(2)}\right)$ to the case in which one considers more than two pure states $\psi^{(i)}$.

Given a set of states $\left\{\psi^{(i)}\right\}_{i=1}^{M}$ and a probability $p$, we

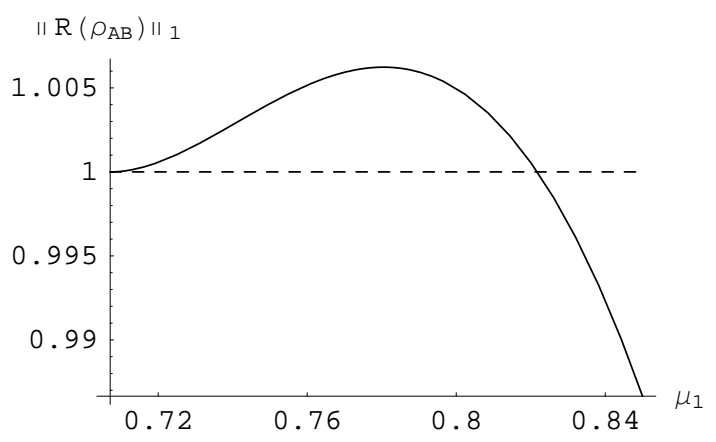

FIG. 3: Comparison of the detection power of realignment and partial transposition in the $d_{1}=d_{2}=2$ case. We take $\psi^{(1)}=\psi^{(2)}=\psi$, where $\psi$ is a pure (entangled) state of two qubits, characterized by its larger Schmidt coefficient $\mu_{1}$. We consider $\left\|R\left(\rho_{A B}\right)\right\|_{1}$, for $\rho_{A B}=\rho_{p_{\Gamma}}(\psi, \psi)$, i.e. for the state at the border of PPT states. For most of the range $1 / \sqrt{2} \leq \mu_{1} \leq 1$ partial transposition is more sensitive than realignment, i.e. $\left\|R\left(\rho_{A B}\right)\right\|_{1}<1$ even if a slight change of $p$ makes the state NPT entangled. Anyway the plot shows that realignment is more sensitive than partial transposition for $\psi$ almost maximally entangled, i.e $\left\|R\left(\rho_{A B}\right)\right\|_{1}>1$ even if the state is PPT.

\begin{tabular}{|c|c|}
\hline \hline$\psi^{(1)}, \psi^{(2)}$ & $\rho_{p}\left(\psi^{(1)}, \psi^{(2)}\right)$ \\
\hline \hline both separable & separable for all $0 \leq p \leq 1$ \\
\hline one entangled & NPT entangled for all $0 \leq p \leq 1$ \\
\hline both entangled & $\begin{array}{c}\mu^{(1)} \neq \mu^{(2)}: \text { PPT entangled for } 0<p<p_{\Gamma} \\
\mu^{(1)}=\mu^{(2)}: \text { no general statement }\end{array}$ \\
\hline
\end{tabular}

TABLE I: Relation between the entanglement properties of the two pure states $\psi^{(1)}, \psi^{(2)}$, and those of $\rho_{p}\left(\psi^{(1)}, \psi^{(2)}\right)$. When both $\psi^{(1)}, \psi^{(2)}$ are entangled, and do not have the same Schmidt coefficients (i.e., they are not equivalent up to local unitaries), $\rho_{p}\left(\psi^{(1)}, \psi^{(2)}\right)$ is PPT entangled in the interval $0<p<p_{\Gamma}$. If both the pure states are entangled, but $\mu^{(1)}=\mu^{(2)}$, the techniques (witnesses) adopted in this work do not help. There are choices of $\psi^{(1)}, \psi^{(2)}$ such that the mixed state $\rho_{p}\left(\psi^{(1)}, \psi^{(2)}\right)$ is separable as soon as, decreasing $p$, it is PPT (see Section VI), as well as other choices such that the corresponding mixed states can be PPT entangled (see Fig. 3).

define:

$$
\rho_{p}\left(\left\{\psi^{(i)}\right\}\right)=(1-p) \bigotimes_{i=1}^{M} \frac{\mathbb{1}-P_{\psi^{(i)}}}{d_{i}^{2}-1}+p \bigotimes_{i=1}^{M} P_{\psi^{(i)}} .
$$

To prove that for $M \geq 3$ the state is entangled for $p>0$ as soon as one of the $\psi^{(i)}$ is entangled, it is sufficient to use the class of witnesses we studied for $M=2$.

Indeed, for $M \geq 3$ it is always possible to split any set of natural numbers $\left\{r^{(i)}\right\}_{i=1}^{M}$ into two non-empty disjoint sets, which w.l.o.g can be indicated as $\left\{r^{(i)}\right\}_{i=1}^{m}$ and $\left\{r^{(i)}\right\}_{i=m+1}^{M}$, and such that $\prod_{i=1}^{m} r^{(i)} \neq \prod_{i=m+1}^{M} r^{(i)}$. Let us consider the case in which the numbers $\left\{r^{(i)}\right\}_{i=1}^{M}$ are 
the Schmidt ranks of the states in $\left\{\psi^{(i)}\right\}_{i=1}^{M}$. For the sake of testing entanglement, it is possible to consider two states $\left|\tilde{\psi}^{(1)}\right\rangle=\bigotimes_{i=1}^{m} \psi^{(i)}$ and $\left|\tilde{\psi}^{(2)}\right\rangle=\bigotimes_{i=m+1}^{M} \psi^{(i)}$ of different Schmidt rank (which is a multiplicative quantity under tensoring). Thus, if at least one state $\psi^{(i)}$ is entangled, we can construct a non-trivial entanglement witness $W_{\epsilon}\left(\tilde{\psi}^{(1)}, \tilde{\psi}^{(2)}\right), \epsilon>0$, as in (15) such that $\operatorname{Tr}\left(\rho_{p}\left(\left\{\psi^{(i)}\right\} W_{\epsilon}\left(\tilde{\psi}^{(1)}, \tilde{\psi}^{(2)}\right)\right)=-p \epsilon\right.$. Notice that, if all states $\psi^{(i)}$ are separable, then also $\rho_{p}\left(\left\{\psi^{(i)}\right\}\right)$ is separable, i.e. there is no entanglement to be detected.

Similarly to the case $M=2$, it is possible to prove that the smallest eigenvalue of a state (26) corresponds to a Schmidt rank 2 eigenvector, so that as soon as the state is NPT we know also it is distillable. Moreover, it is possible to find a $p>0$, such that the state $\rho_{p}\left(\left\{\psi^{(i)}\right\}\right)$ is PPT entangled, if and only if the states $\left\{\psi^{(i)}\right\}_{i=1}^{M}$ are all entangled.

\section{A. Maximally entangled pure states $\psi^{(i)}$}

We now focus on a even more specific class of states. Recalling the definition (6) of maximally entangled state $P_{d}^{+}$, we define the states

$$
\rho_{p}\left(d_{1}, \ldots, d_{M}\right)=(1-p) \bigotimes_{i=1}^{M} \frac{\mathbb{1}-P_{d_{i}}^{+}}{d_{i}^{2}-1}+p \bigotimes_{i=1}^{M} P_{d_{i}}^{+} .
$$

Compare them to the isotropic states for a $d \otimes d$ system:

$$
\rho_{p}(d)=(1-p) \frac{\left(\mathbb{1}-P_{d}^{+}\right)}{d^{2}-1}+p P_{d}^{+}
$$

Isotropic states can be considered a subclass of the class of states we are studying, with $M=1$. It is remarkable that isotropic states $\rho_{p}(d)$ are either distillable or separable: no phenomenon of bound entanglement (either PPT or NPT - if existing) is present in such class, while it is sufficient to go to $M=2$ to have it.

It is worth noticing that $\rho_{p}(d, d)$ is separable for all values of $p$ for which it is PPT, i.e for $0 \leq p \leq 1 / d^{2}$ [37], and indeed we are not able to construct a witness of the form (15) for it, since in this case $\psi^{(1)}$ and $\psi^{(2)}$ have the same Schmidt coefficients - they are equal. On the other hand, a witness as in (15) exists for $\rho_{p}\left(d_{1}, d_{2}\right)$ in the case $d_{2}>d_{1} \geq 2$.

As regards the sensitivity of partial transposition, for $\rho_{p}\left(d_{1}, \ldots, d_{M}\right)$ we have

$$
p_{\Gamma}=\frac{1}{1+\left(d_{M}-1\right) \prod_{i=1}^{M-1}\left(d_{i}+1\right)},
$$

taking w.l.o.g. $d_{1} \leq d_{2} \leq \ldots \leq d_{M}$. We have seen that, if $M=2$ and we consider the case in which both pure states $\psi^{(i)}$ are maximally entangled in dimension $d_{i}, \mathrm{PT}$ is always more sensitive than realignment. To study the more general case $M>2$, we restrict ourselves, for simplicity, to the case in which all the dimensions $d_{i}$ coincide $\left(d_{i}=d \geq 2\right)$ and $\psi^{(i)}=\Psi_{d}^{+}$for all $i=1, \ldots M$ :

$$
\rho_{p}(d ; M) \equiv \rho_{p}(\underbrace{d, d, \ldots, d}_{\mathrm{M} \text { times }}) .
$$

In this case we have that $\rho_{p}(d ; M)$ is $\mathrm{PPT}$ for $p \leq p_{\Gamma}=$ $\frac{1}{1+(d-1)(d+1)^{M-1}}$. As regards realignment, we have

$$
\left\|R\left(\rho_{p}(d ; M)\right)\right\|_{1}=\frac{1}{d^{M}} \sum_{j=0}^{M}\left(\begin{array}{c}
M \\
j
\end{array}\right)\left|1-p-p\left(1-d^{2}\right)^{j}\right|
$$

While it is not trivial to find an analytical solution in $p$ of the inequality $\left\|R\left(\rho_{p}(d ; M)\right)\right\|_{1} \leq 1$, it is possible to see that there are cases in which the realignment criterion is more sensitive than PT. Indeed, this happens for $d=2$ and $M \geq 3$ odd. To verify this, it is sufficient to plug in (30) the corresponding value of $p_{\Gamma}$, i.e. $p=p_{\Gamma}=\frac{1}{1+3^{M-1}}$. By definition, for such value of $p$ the state is PPT and the condition $\left\|R\left(\rho_{p_{\Gamma}}(d ; M)\right)\right\|_{1}>1$ is satisfied for all odd values of $M \geq 3$, while $\left\|R\left(\rho_{p_{\Gamma}}(d ; M)\right)\right\|_{1}=1$ for $M=1$ and $M$ even. Numerical results indicate that $d=2$ and $M \geq 3$ odd is the only case in which realignment detects PPT entangled states of the form $\rho_{p}(d ; M)$, but we could not verify this analytically.

\section{MORE ON WITNESSES}

We proceed now to some remarks as regards the witnesses we analyzed.

\section{A. Simplified witnesses}

We have seen that the necessary and sufficient condition to have a non-trivial entanglement witness $W_{\epsilon}\left(\psi^{(1)}, \psi^{(2)}\right)$, with $\epsilon>0$, is that the states $\psi^{(i)}$ have different Schmidt coefficients. When the Schmidt ranks of states $\psi^{(i)}$ are different, i.e. w.l.o.g. $r_{1}<r_{2}$, it is possible to detect the entanglement of $\rho_{p}\left(\psi^{(1)}, \psi^{(2)}\right)$ by means of a witness with a structure even simpler than that of $W_{\epsilon}\left(\psi^{(1)}, \psi^{(2)}\right)$. In such a case, in fact, it is possible to consider non trivial $(\epsilon>0)$ witnesses of the form

$$
\begin{aligned}
\tilde{W}_{\epsilon}\left(\psi^{(1)}, \psi^{(2)}\right) & =P_{\psi^{(1)}} \otimes\left(\mathbb{1}-(1+\epsilon) P_{\psi^{(2)}}\right) \\
& =P_{\psi^{(1)}} \otimes \mathbb{1}-(1+\epsilon) P_{\psi^{(1)}} \otimes P_{\psi^{(2)}} .
\end{aligned}
$$

For this choice,

$$
\begin{aligned}
& \left\langle\alpha_{A} \otimes \beta_{B}\left|\tilde{W}_{\epsilon}\left(\psi^{(1)}, \psi^{(2)}\right)\right| \alpha_{A} \otimes \beta_{B}\right\rangle= \\
& \operatorname{Tr}\left(\left(\beta^{T} \mu^{(1)} \alpha\right)^{\dagger}\left(\beta^{T} \mu^{(1)} \alpha\right)\right)-(1+\epsilon)\left|\operatorname{Tr}\left(\mu^{(2)} \beta^{T} \mu^{(1)} \alpha\right)\right|^{2} .
\end{aligned}
$$

Following the same reasoning we used for $W_{\epsilon}\left(\psi^{(1)}, \psi^{(2)}\right)$, we see that the quantity (32) can be made negative for 
any $\epsilon>0$ if and only if (w.l.o.g) there exist $\alpha$ and $\beta$ such that

$$
\mu^{(2)}=\beta^{T} \mu^{(1)} \alpha .
$$

This is possible if and only if the rank of $\mu^{(1)}$ is greater than that of $\mu^{(2)}$.

We may better understand this result by considering that

$$
\begin{aligned}
& \tilde{W}_{\epsilon}\left(\psi^{(1)}, \psi^{(2)}\right) \\
= & \left(P_{\psi^{(1)}} \otimes \mathbb{1}\right) \circ\left(\mathbb{1} \otimes\left(\mathbb{1}-(1+\epsilon) P_{\psi^{(2)}}\right)\right) \circ\left(P_{\psi^{(1)}} \otimes \mathbb{1}\right)
\end{aligned}
$$

and that

$$
\left(P_{\psi^{(1)}} \otimes \mathbb{1}\right)\left|\alpha_{A} \otimes \beta_{B}\right\rangle=\left|\psi^{(1)}\right\rangle \otimes|\gamma\rangle,
$$

with $|\gamma\rangle=\sum_{i} \mu_{i}^{(1)}\left(\sum_{l} \alpha_{i l}|l\rangle\right) \otimes\left(\sum_{k} \beta_{i k}|k\rangle\right)$. It is clear that, by the right choice of $\alpha$ and $\beta, \gamma$ - though in general not normalized - can be made proportional to any state whose Schmidt rank is not greater than the one of $\psi^{(1)}$. In particular, if $\psi^{(2)}$ has the same Schmidt rank that $\psi^{(1)}$ has, it is possible to choose $\alpha$ and $\beta$ such that

$$
\left\langle\alpha \beta\left|\tilde{W}_{\epsilon}\left(\psi^{(1)}, \psi^{(2)}\right)\right| \alpha \beta\right\rangle=-\epsilon|c|^{2},
$$

with $|\gamma\rangle=c\left|\psi^{(2)}\right\rangle$ and $|c|>0$. Therefore, in this case, $\tilde{W}$ is positive on separable states if and only if $\epsilon=0$.

Notice that in Section VI] when analyzing the multistate case for $M \geq 3$, we argued that as soon as one state $\psi^{(i)}$ is entangled, it is possible to consider two states $\left|\tilde{\psi}^{(j)}\right\rangle, j=1,2$ of different rank obtained from $\psi^{(i)}$ s by tensoring. It is therefore clear that for $M \geq 3$, as soon as the problem is not trivial (i.e. not all the states $\psi^{(i)}$ are factorized), it is always possible to consider a witness $\tilde{W}_{\epsilon}\left(\tilde{\psi}^{(1)}, \tilde{\psi}^{(2)}\right)$ of the form (31).

\section{B. Canonical witnesses for $\rho_{p}\left(\left\{\psi^{(i)}\right\}\right)$}

Both for $W=W_{\epsilon}\left(\psi^{(1)}, \psi^{(2)}\right)$ (Eq. (15)) and $W=\tilde{W}_{\epsilon}\left(\psi^{(1)}, \psi^{(2)}\right)$ (Eq. (31)), we have not only $\operatorname{Tr}\left(W \rho_{0}\left(\psi^{(1)}, \psi^{(2)}\right)\right)=0$, but, more strongly,

$$
W \rho_{0}\left(\psi^{(1)}, \psi^{(2)}\right)=\rho_{0}\left(\psi^{(1)}, \psi^{(2)}\right) W=0,
$$

i.e. the witnesses [38] are orthogonal to the separable part - which corresponds to $\rho_{0}\left(\psi^{(1)}, \psi^{(2)}\right)$ - of a state $\rho_{p}\left(\psi^{(1)}, \psi^{(2)}\right)$. Indeed, we have $\tilde{W}_{\epsilon}\left(\psi^{(1)}, \psi^{(2)}\right) \leq$ $W_{\epsilon}\left(\psi^{(1)}, \psi^{(2)}\right)$ (compare (15) and (31) ) and

$$
\begin{aligned}
W_{\epsilon}\left(\psi^{(1)}, \psi^{(2)}\right) & =\mathbb{1}-\left(\mathbb{1}-P_{\psi^{(1)}}\right) \otimes\left(\mathbb{1}-P_{\psi^{(2)}}\right) \\
& -\epsilon P_{\psi^{(1)}} \otimes P_{\psi^{(2)}} .
\end{aligned}
$$

Moreover, $\rho_{0}\left(\psi^{(1)}, \psi^{(2)}\right)$ is exactly defined as the state corresponding (via normalization) to the projector $(\mathbb{1}-$ $\left.P_{\psi^{(1)}}\right) \otimes\left(\mathbb{1}-P_{\psi^{(2)}}\right)$.
In the case of $M \geq 3$ states $\psi^{(i)}$, we argued (see Section VI. paragraph following Eq. (26)) that, as soon as one state $\psi^{(i)}$ is entangled, there exists a non-trivial entanglement witness of the form $W_{\epsilon}\left(\tilde{\psi}^{(1)}, \tilde{\psi}^{(2)}\right)$ which detects the entanglement of $\rho_{p}\left(\left\{\psi^{(i)}\right\}\right)$. The states $\tilde{\psi}^{(i)}, i=1,2$ were taken to be tensor products of two disjoint subsets of $\left\{\psi^{(i)}\right\}$, so that $\left|\tilde{\psi}^{(1)}\right\rangle \otimes\left|\tilde{\psi}^{(2)}\right\rangle=\bigotimes_{i=1}^{M}\left|\psi^{(i)}\right\rangle$. We can instead consider a witness of the form

$$
\begin{aligned}
W_{\epsilon}\left(\left\{\psi^{(i)}\right\}\right) & =\mathbb{1}-\bigotimes_{i=1}^{M}\left(\mathbb{1}-P_{\psi^{(i)}}\right) \\
& -\epsilon \bigotimes_{i=1}^{M} P_{\psi^{(i)}} .
\end{aligned}
$$

We have $W_{\epsilon}\left(\left\{\psi^{(i)}\right\}\right) \geq W_{\epsilon}\left(\tilde{\psi}^{(1)}, \tilde{\psi}^{(2)}\right)$, but $W_{\epsilon}\left(\left\{\psi^{(i)}\right\}\right)$ has the same expectation value $-\epsilon p$ with respect to the states $\rho_{p}\left(\left\{\psi^{(i)}\right\}\right)$. Moreover, it can be considered as a modification of the projector onto the subspace orthogonal to the support of the separable part $\rho_{0}\left(\left\{\psi^{(i)}\right\}\right)$ of the state, with the modification $-\epsilon \bigotimes_{i=1}^{M} P_{\psi^{(i)}}$ tailored to "intercept" the entangled part of the state. Notice that the witness $W_{\epsilon}\left(\left\{\psi^{(i)}\right\}\right)$ depends only on $\epsilon$ and on the set $\left\{\psi^{(i)}\right\}$, not on the choice of two subsets of $\left\{\psi^{(i)}\right\}$, unlike $W_{\epsilon}\left(\tilde{\psi}^{(1)}, \tilde{\psi}^{(2)}\right)$.

\section{MULTIPARTITE CASE}

Now we consider the multipartite case, i.e. the states $\psi^{(i)}, i=1 \ldots, M$, are states of $N$ parties. From the results presented in Section $\mathrm{V}$ we know that the state $\rho_{p}\left(\left\{\psi^{(i)}\right\}\right)$ can be made PPT, with respect to a given bipartite cut $S_{1}: S_{2}$, for some strictly positive $p$ only if all the states $\psi^{(i)}$ are entangled with respect to that cut. Therefore, for this to happen for any possible bipartite cut, all the states $\psi^{(i)}$ must be $N$-partite entangled.

As regards witnesses, we are able to provide a nontrivial (i.e. not positive semidefinite) witness which detects bipartite $S_{1}: S_{2}$ entanglement, if (i) $M=2$ and the states $\psi^{(1)}$ and $\psi^{(1)}$ have different Schmidt coefficients with respect to the cut, or (ii) $M \geq 3$ and at least one state $\psi^{(i)}$ is entangled with respect to the cut. As we discussed in Section VIIB, it is always possible to consider witnesses $W_{\epsilon}\left(\left\{\psi^{(i)}\right\}\right)$ of the form (35), for every cut. In the construction of such witnesses, the only parameter dependent from the cut is $\epsilon$. If, for a given cut, one of the just mentioned conditions (i) and (ii) is valid, then it is possible to take $\epsilon>0$ and detect bipartite entanglement by means of the corresponding witness. Let us consider

$\tilde{\epsilon}=\min _{S_{1}: S_{2}} \max \left\{\epsilon \mid\left\langle\alpha_{S_{1}} \otimes \beta_{S_{2}}\left|W_{\epsilon}\left(\left\{\psi^{(i)}\right\}\right)\right| \alpha_{S_{1}} \otimes \beta_{S_{2}}\right\rangle \geq 0\right\}$.

If $\tilde{\epsilon}>0$, then $W_{\tilde{\epsilon}}\left(\left\{\psi^{(i)}\right\}\right)$ is a nontrivial witness for genuine multipartite entanglement. Thus, we get immediately that $\rho_{p}\left(\left\{\psi^{(i)}\right\}\right)$ is $N$-partite entangled for every $p>0$, since its entanglement is detected by a witness which is positive with respect to all biseparable states. 
Thus, it is possible to decide to construct a state which is PPT with respect to some desired bipartitions, and NPT with respect to the remaining ones. To do so, it is sufficient to choose opportunely the states $\left\{\psi^{(i)}\right\}$ and $p$. Indeed, the mixed state is NPT with respect to a bipartition $S_{1}: S_{2}$ for all $p>0$ if and only if there is a state $\psi_{i}^{(i)}$ which is $S_{1}: S_{2}$ separable. If the states satisfy (i) or (ii) for every cut, the mixed state $\rho_{p}\left(\left\{\psi^{(i)}\right\}\right)$ for sure contains $N$-partite entanglement (for $p>0$ ), because there is a witness which detects it.

\section{TENSOR-LIKE WITNESSES, $k$-POSITIVE MAPS AND NONDECOMPOSABILITY}

Building upon the considerations of Section VIIA we discuss here the possibility of obtaining nondecomposable witnesses able to detect PPT bound entangled states, by composing through tensor product a decomposable witness (unable to detect PPT bound entanglement) with a positive operator (w.l.o.g. a state).

Lemma III.1 of [39] says that, if a state $\sigma$ on $\mathcal{H}_{A_{1}} \otimes \mathcal{H}_{B_{1}}$ has Schmidt number $k$, and $\eta$ is an operator on $\mathcal{H}_{A_{2}} \otimes \mathcal{H}_{B_{2}}$ which is positive with respect to states of Schmidt number $k l$, then $\sigma \otimes \eta$ is positive with respect to states $\tau$ on $\mathcal{H}_{A} \otimes \mathcal{H}_{B}$ of Schmidt number $l$, i.e. $\operatorname{Tr}((\sigma \otimes \eta) \tau) \geq 0$. The proof of such Lemma [39] is a generalization of the reasoning we have adopted in Section VII A. In particular, it is sufficient to consider the case $\sigma=|\phi\rangle\langle\phi|$ and $\tau=|\psi\rangle\langle\psi|$, and notice that

$$
\begin{aligned}
\operatorname{Tr}(|\psi\rangle\langle\psi|(|\phi\rangle\langle\phi| \otimes \eta)) & =\operatorname{Tr}_{2}\left(\operatorname{Tr}_{1}(|\psi\rangle\langle\psi|(|\phi\rangle\langle\phi| \otimes \mathbb{1})) \eta\right) \\
& =\langle\gamma|\eta| \gamma\rangle,
\end{aligned}
$$

with $(|\phi\rangle\langle\phi| \otimes \mathbb{1})|\psi\rangle=|\phi\rangle \otimes|\gamma\rangle$. Considering that $\psi$ has Schmidt rank $l$, and that the action of $(|\phi\rangle\langle\phi| \otimes \mathbb{1})$ on a separable states can create at most a state of Schmidt rank $k$ (see (34)), we conclude that the state $\gamma$ has at most Schmidt rank $k l$. Note that $\gamma$ is not normalized, in general.

We are interested in operators that are entanglement witnesses, i.e. such that they are positive on separable states. We correspondingly take $l=1$, and consider a state $\sigma=|\phi\rangle\langle\phi|=P_{\phi}$ of Schmidt number $k$ and a Schmidt-rank $m$ witness $W$, with $m \geq k+1$. We compose them to give an operator $P_{\phi} \otimes W$, which is then positive on separable states by construction, according to the Lemma III.1 of [39]. If (i) $\phi$ is entangled (i.e. $k \geq 2$ ) and (ii) $\psi_{W}$, of Schmidt rank strictly greater than $k$, is such that $\left\langle\psi_{W}|W| \psi_{W}\right\rangle<0$, then $P_{\phi} \otimes W$ is a non-decomposable entanglement witness. Indeed, we can consider $p>0$ such that $\rho_{p}\left(\phi, \psi_{W}\right)$ is PPT - both $\phi$ and $\psi_{W}$ are entangled - and have

$$
\operatorname{Tr}\left(P_{\phi} \otimes W \rho_{p}\left(\phi, \psi_{W}\right)\right)=p\left\langle\psi_{W}|W| \psi_{W}\right\rangle<0 .
$$

With these considerations and exploiting the ChoiJamiołkowski isomorphism, it is immediate to state a theorem relating the properties of $k$-positivity, complete positivity and decomposability of maps.

Theorem 1. A linear map $\Lambda$ which is $k$-positive, $k \geq 2$, is completely positive if and only if $\mathrm{id}_{k} \otimes \Lambda$ is decomposable.

Proof. The only if part is trivial: if $\Lambda$ is CP, then $\mathrm{id}_{k} \otimes \Lambda$ is trivially decomposable - it is $\mathrm{CP}$ itself. To prove the if part, let us suppose that $\Lambda$ is not $\mathrm{CP}$ and show that $\operatorname{id}_{k} \otimes \Lambda$ is nondecomposable. Indeed, if $\Lambda$ is not $\mathrm{CP}$, then, even though the corresponding witness $W_{\Lambda}$ is positive on Schmidt rank $k$ states [28, 31], there exists a Schmidt rank $m$ state $\psi_{W_{\Lambda}}, m>k$, such that $\left\langle\psi_{W_{\Lambda}}\left|W_{\Lambda}\right| \psi_{W_{\Lambda}}\right\rangle<$ 0 . Thus, as remarked before, the witness $P_{k}^{+} \otimes W$ is nondecomposable and the same holds for its isomorphic $\operatorname{map~id}_{k} \otimes \Lambda$.

Notice that in the theorem we could have used $\operatorname{id}_{l} \otimes \Lambda$, with any $2 \leq l \leq k$, instead of $\operatorname{id}_{k} \otimes \Lambda$.

The results just exposed imply that, for $k \geq 2$, as soon as we know that a PnCP map $\Lambda$ is $k$-positive or that a non-positive witness $W$ is positive on Schmidtnumber $k$ states, we know that, for example, $\operatorname{id}_{k} \otimes \Lambda$ and $P_{k}^{+} \otimes W$ are respectively a positive nondecomposable map and a nondecomposable witness, without caring about the decomposability of $\Lambda$ or $W$.

We provide now a simple example illustrating how to pass from provably decomposable witnesses to nondecomposable witnesses through tensoring. Obviously, the example could be recast immediately in terms of maps.

Let us consider witnesses of the form

$$
W_{\epsilon}(\psi)=\mathbb{1}-(1+\epsilon)|\psi\rangle\langle\psi| .
$$

If we consider expectation values with respect to pure states we obtain

$$
\left\langle\varphi\left|W_{\epsilon}(\psi)\right| \varphi\right\rangle=1-(1+\epsilon)|\langle\psi \mid \varphi\rangle|^{2} .
$$

Suppose $\psi$ has Schmidt rank $r$ and Schmidt decomposition $|\psi\rangle=\sum_{i=1}^{r} \mu_{i}|i i\rangle$. It can be proved by Lagrange multipliers that

$$
\max _{\{\varphi \text { s.t. } S R(\phi) \leq k\}}|\langle\psi \mid \varphi\rangle|^{2}=\sum_{i=1}^{k} \mu_{i}^{2},
$$

where the maximum is taken with respect to states $\varphi$ which have at most Schmidt rank $k \leq r$, and the Schmidt coefficients of $\psi$ are ordered as $\mu_{i} \geq \mu_{i+1}$. Thus, if we want the witness $W_{\epsilon}(\psi)$ to be positive on states of Schmidt rank $k$, we must have

$$
\epsilon \leq \frac{1-\sum_{i=1}^{k} \mu_{i}^{2}}{\sum_{i=1}^{k} \mu_{i}^{2}} .
$$

Notice that if $k=r$ we must put $\epsilon=0$. Correspondingly, for $k<r$ (in the case $r \geq 2$ ), let us define the Schmidt number $k+1$ witness

$$
W_{k}(\psi)=\mathbb{1}-\frac{1}{\sum_{i=1}^{k} \mu_{i}^{2}}|\psi\rangle\langle\psi| .
$$


Among $W_{k}(\psi)$ 's, for fixed $\psi$, the witness able to detect the largest number of entangled states is of course $W_{1}(\psi)$. Yet, $W_{1}(\psi)$ cannot detect any PPT bound entangled state, as we now prove.

Every pure state $\psi$ in $\mathbb{C}^{d} \otimes \mathbb{C}^{d}$ can be written as $|\psi\rangle=\sqrt{d}(A \otimes \mathbb{1})\left|\Psi_{d}^{+}\right\rangle$, with $\operatorname{Tr}\left(A^{\dagger} A\right)=1$. The Schmidt coefficients of $\psi$ are given by the singular values of $A$. In particular, for the largest Schmidt coefficient we have $\mu_{1}=\|A\|_{\infty}=\sqrt{\left\|A A^{\dagger}\right\|_{\infty}}$, where $\|X\|_{\infty}$ is the operator norm of $X$. We have $(|\psi\rangle\langle\psi|)^{\Gamma}=A \otimes \mathbb{1} V A^{\dagger} \otimes \mathbb{1}$, with $V$ the swap operator. Notice that $V \leq \mathbb{1}$.

The witness $W_{1}(\psi)$, does not detect a state $\rho$ as entangled if and only if $\langle\psi|\rho| \psi\rangle \leq \mu_{1}^{2}$. If $\rho=\rho_{A B}$ is PPT, $\tilde{\rho}=\rho^{\Gamma}$ is a normalized state and

$$
\begin{aligned}
\langle\psi|\rho| \psi\rangle & =\operatorname{Tr}\left(\rho^{\Gamma}(|\psi\rangle\langle\psi|)^{\Gamma}\right) \\
& =\operatorname{Tr}\left(\tilde{\rho}(A \otimes \mathbb{1}) V\left(A^{\dagger} \otimes \mathbb{1}\right)\right) \\
& \leq \operatorname{Tr}\left(\tilde{\rho}\left(A A^{\dagger} \otimes \mathbb{1}\right)\right) \\
& =\operatorname{Tr}\left(\tilde{\rho}_{A} A A^{\dagger}\right) \\
& \leq \max _{\phi}\left\langle\phi\left|A A^{\dagger}\right| \phi\right\rangle \\
& =\mu_{1}^{2},
\end{aligned}
$$

where $\tilde{\rho}_{A}=\operatorname{Tr}_{B}(\tilde{\rho})$. A straightforward proof can also be obtained by considering that the reduction criterion [7] is weaker than the PT criterion. Thus, for every PPT state $\rho=\rho_{A B}$ we have $\rho \leq \rho_{A} \otimes \mathbb{1}$. For the state $\psi$ under consideration:

$$
\langle\psi|\rho| \psi\rangle \leq\left\langle\psi\left|\rho_{A} \otimes \mathbb{1}\right| \psi\right\rangle=\operatorname{Tr}\left(\rho_{A} A A^{\dagger}\right) \leq \mu_{1}^{2} .
$$

Witnesses $W_{k}(\psi), N \geq 2$ are even worse in detecting PPT entangled states. Yet, for every $2 \leq k \leq r-1$, and for any state $\phi$ with Schmidt rank $2 \leq l \leq k, P_{\phi} \otimes W_{k}(\psi)$ is a nondecomposable entanglement witness.

\section{CONCLUSIONS}

We have been able to prove that any state $\rho_{p}\left(\left\{\psi^{(i)}\right\}\right)$ is entangled as soon as $p>0$, for any set $\left\{\psi_{(i)}\right\}_{i=1}^{M}$, when at least one state $\psi_{(i)}$ is entangled, except in the case with only two pure states $\psi^{(i)}$ with the same Schmidt coefficients. In the latter case the state could be entangled as well, but an entanglement witness different from (15) would be required to prove it.

The structure of the states $\rho_{p}\left(\left\{\psi^{(i)}\right\}\right)$ is very simple: all the entanglement appears to be concentrated in an eigenvector of the mixed state, while the separable part, in the suitable region of parameters, plays the role of a "cover", which prevents the detection by partial transposition (and hence distillation). We remark the resemblance of the class of states with isotropic states, most evident when considering the special case of maximally entangled pure states $\left\{\psi^{(i)}=\Psi_{d_{i}}^{+}\right\}$. Indeed, isotropic states were involved in the analysis which signed the first appearance of the class of states $\rho_{p}\left(\psi^{(1)}, \psi^{(2)}\right)$ in literature [18].
Our analysis differs from the one appearing in [18] and [19], because we focus on the properties of the states, rather than on what they allow to do, and we construct witnesses to detect entanglement both in the bipartite and multipartite settings. Moreover, we generalize the states to the case of the possible choice of many pure states, i.e. from the states $\rho_{p}\left(\psi^{(1)}, \psi^{(2)}\right)$ to the states $\rho_{p}\left(\left\{\psi^{(i)}\right\}\right)$. It is clear that the states $\rho_{p}\left(\left\{\psi^{(i)}\right\}\right)$ can be modified, both in their separable and entangled parts, to provide larger classes of PPT entangled states. Indeed, the key point toward the construction of PPT entangled states, is that the separable part of $\rho_{p}\left(\left\{\psi^{(i)}\right\}\right)$ is not only positive semidefinite under partial transposition, but strictly positive, so that slightly changing it does not affect the positivity condition. For example, using states similar to the ones studied in this paper, it is possible to prove that many of the positive maps that were conjectured to be nondecomposable in [24], are actually so [40]. One remarkable property of the structure (26) is that states $\rho_{p}\left(\left\{\psi^{(i)}\right\}\right)$ are completely characterized by a set of pure states $\left\{\psi^{(i)}\right\}$ and a mixing parameter $p$. Moreover, they are separable for $p=0$ and entangled - for almost any choice of $\left\{\psi^{(i)}\right\}$ with at least one $\psi^{(i)}$ entangled - for $p>0$. We hope that the variety of parameters at disposal through the choice of the set of pure states $\left\{\psi^{(i)}\right\}$ (and of the mixing parameter $p$ ), could lead to the study of interesting cases/effects both in the bipartite and in multipartite setting. Moreover, the simplicity of the structure of this class of states suggests that they might be used to offer the first experimental verification of the existence, and properties, of bound entanglement. Preliminary studies of the robustness of these states under the action of noise, for some proper choice of the two pure states $\psi^{(1)}$ and $\psi^{(2)}$, confirms the fact that experimental construction of these states should be possible with current technology. A detailed analysis of the noise tolerance of the states $\rho_{p}$, together with a study of the possible experimental realization of these states will be presented elsewhere.

Notice that all the results regarding witnesses can be directly translated into results regarding maps through the Choi-Jamiołkowski isomorphism, so that in this paper we provide many examples of nondecomposable maps, useful to detect the entanglement of PPT states. Moreover, through the analysis of states $\rho_{p}\left(\left\{\psi^{(i)}\right\}\right)$ and of the corresponding witnesses, we could provide a relationship among the properties of $k$-positivity, complete positivity and decomposability: any map that is $k$-positive, for $k \geq 2$, but not completely positive, can be extended to a nondecomposable map. Thus, it seems that further analysis of the property of $k$-positivity could not only be useful to study the Schmidt-number property of states, following [28], but the entanglement property itself.

We thank H.J. Briegel, O. Guehne, M. Horodecki, P. Horodecki, R. Horodecki, A. Kossakowski and W. A. Majewski for discussions. We gratefully acknowledge support by Austrian Science Foundation (FWF), EU (RESQ (IST 2001 37559), OLAQUI, IP SCALA). 
[1] M. A. Nielsen and I. L. Chuang, Quantum Computation and Quantum Information (Cambridge University Press, Cambridge, 2000).

[2] G. Alber, T. Beth, M. Horodecki, P. Horodecki, R. Horodecki, M. Rotteler, H. Weinfurter, R. Werner, and A. Zeilinger, Quantum Information: An Introduction to Basic Theoretical Concepts and Experiments (Springer, 2001).

[3] C. H. Bennett, G. Brassard, C. Crepeau, R. Jozsa, A. Peres, and W. K. Wootters, Phys. Rev. Lett 70, 1895 (1983).

[4] C. Bennett and S. Wiesner, Phys. Rev. Lett 69, 20 (1992).

[5] R. Werner, Phys. Rev. A 40, 4277 (1989).

[6] W. Dür, G. Vidal, and J. I. Cirac, Phys. Rev. A 62, 062314 (2000), quant-ph/0005115.

[7] M. Horodecki and P. Horodecki, Phys. Rev. A 59, 4206 (1999), quant-ph/9708015.

[8] A. Peres, Phys. Rev. Lett. 77, 1413 (1996).

[9] M. Horodecki, P. Horodecki, and R. Horodecki, Phys. Lett. A 223, 1 (1996), quant-ph/9605038.

[10] O. Rudolph, quant-ph/0202121.

[11] K. Chen, L.-A. Wu, and L. Yang, quant-ph/0205017.

[12] M. Horodecki, P. Horodecki, and R. Horodecki, Open Syst. Inf. Dyn. 13, 103 (2006).

[13] P. Wocjan and M. Horodecki, Open Syst. Inf. Dyn. 12, 331 (2005).

[14] M. Choi, Linear Algebr. Appl. 10, 285 (1975).

[15] A. Jamiołkowski, Rep. Math. Phys. 3, 275 (1972).

[16] L. Masanes, PRL 96, 150501 (2006).

[17] P. Horodecki, M. Horodecki, and R. Horodecki, Phys. Rev. Lett 82, 1056 (1999), quant-ph/9806058.

[18] S. Ishizaka, Phys. Rev. Lett 93, 190501 (2004), quantph/0403016.

[19] S. Ishizaka and M. Plenio, Phys. Rev. A 71, 052303 (2005).

[20] P. Horodecki, Phys. Lett. A 232, 333 (1997).

[21] P. Horodecki and M. Lewenstein, Phys. Rev. Lett 85, 2657 (2000).

[22] D. Bruss and A. Peres, Phys. Rev. A 61, 30301(R) (2000), quant-ph/9911056.

[23] S. Yu and N. Liu, Phys. Rev. Lett 95, 150504 (2005).

[24] M. Piani, Phys. Rev. A 73, 012345 (2006).
[25] X. Q. L.-J. S. M. Fei and B. Z. Sun, Phys. Lett. A 352, 321 (2006).

[26] D. Chruściński and A. Kossakowski, quant-ph/0602076.

[27] C. H. Bennett, D. P. DiVincenzo, T. Mor, P. W. Shor, J. A. Smolin, and B. M. Terhal, Phys. Rev. Lett 82, 5385 (1999).

[28] B. Terhal and P. Horodecki, Phys. Rev. A 61, 040301 (2000).

[29] B. M. Terhal, Phys. Lett. A. 271, 319 (2000), quant$\mathrm{ph} / 9911057$.

[30] M. Lewenstein, B. Kraus, J. I. Cirac, and P. Horodecki, Phys. Rev. A 62, 052310 (2000).

[31] A. Sanpera, D. Bruß, and M. Lewenstein, Phys. Rev. A 63, 050301 (2001).

[32] A. Acin, D. Bruss, M. Lewenstein, and A. Sanpera, PRL 87, 040401 (2001).

[33] S. Bandyopadhyay and V. Roychowdhury, PRA 69, 040302 (2004).

[34] The problem can be cast in the form (19) w.l.o.g. because there is no normalization constraint on $|\alpha\rangle$ and $|\beta\rangle$, i.e. on matrices $\alpha$ and $\beta$, and we can use this freedom to get rid of one proportionality constant in discussing the condition.

[35] Given a composite system $A_{1} A_{2}$ : $B_{1} B_{2}$, the realignment operation can be split in realignement with respect to subsystems 1 and 2 (exactly as partial transpostion with respect to $A$ can be split into partial transpostion with respect to $A_{1}$ and $\left.A_{2}\right): \quad R\left(\left|i_{A_{1}} m_{A_{2}} j_{B_{1}} n_{B_{2}}\right\rangle\left\langle k_{A_{1}} p_{A_{2}} l_{B_{1}} q_{B_{2}}\right|\right)=$ $R_{1}\left(\left|i_{A_{1}} j_{B_{1}}\right\rangle\left\langle k_{A_{1}} l_{B_{1}}\right|\right) \otimes R_{2}\left(\left|m_{A_{2}} n_{B_{2}}\right\rangle\left\langle p_{A_{2}} q_{B_{2}}\right|\right)=$ $\left|i_{A_{1}} m_{A_{2}} k_{B_{1}} p_{B_{2}}\right\rangle\left\langle j_{A_{1}} n_{A_{2}} l_{B_{1}} q_{B_{2}}\right|$.

[36] M. Horodecki, P. Horodecki, and R. Horodecki, Phys. Rev. Lett 80, 5239 (1998), quant-ph/9801069.

[37] K. G. H. Vollbrecht and R. F. Werner, Phys. Rev. A 64, 062307 (2001).

[38] Note that, if $\tilde{W}_{\epsilon}\left(\psi^{(1)}, \psi^{(2)}\right)$ is a witness for a given $\epsilon$, then also $W_{\epsilon}\left(\psi^{(1)}, \psi^{(2)}\right)$ is a witness for the same value of $\epsilon$, while the converse is not true: consider for example the case the $\psi^{(i)}$ 's have the same Schmidt rank but not the same Schmidt coefficients.

[39] L. Clarisse, Quant. Inf. Comp. (2006).

[40] M. Piani, in preparation. 